\title{
A DIPOLE ON THE SKY: PREDICTIONS FOR HYPERVELOCITY STARS FROM THE LARGE MAGELLANIC CLOUD
}

\author{
Douglas Boubert and N. Wyn Evans \\ Institute of Astronomy, University of Cambridge, Madingley Road, Cambridge CB3 0HA, UK; d.boubert@ast.cam.ac.uk, nwe@ast.cam.ac.uk \\ Received 2016 May 25; revised 2016 June 6; accepted 2016 June 7; published 2016 June 24
}

\begin{abstract}
We predict the distribution of hypervelocity stars (HVSs) ejected from the Large Magellanic Cloud (LMC), under the assumption that the dwarf galaxy hosts a central massive black hole (MBH). For the majority of stars ejected from the LMC, the orbital velocity of the LMC has contributed a significant fraction of their galactic rest-frame velocity, leading to a dipole density distribution on the sky. We quantify the dipole using spherical harmonic analysis and contrast with the monopole expected for HVSs ejected from the Galactic center (GC). There is a tendril in the density distribution that leads the LMC, which is coincident with the well-known and unexplained clustering of HVSs in the constellations of Leo and Sextans. Our model is falsifiable since it predicts that Gaia will reveal a large density of HVSs in the southern hemisphere.
\end{abstract}

Key words: Galaxy: halo - Galaxy: stellar content - Magellanic Clouds

\section{INTRODUCTION}

A hypervelocity star (HVS) is any star whose velocity in the galactic rest frame exceeds the local escape speed of the Milky Way (MW). Several routes have been suggested for their production, the first being reported by Hills (1988), who theorized that the tidal disruption of a stellar binary during a close encounter with the massive black hole $(\mathrm{MBH})$ at the center of the MW could accelerate one member of the binary, in what is now known as the Hills mechanism.

The first candidate HVS, a B-type star traveling with a galactic rest-frame velocity of $709 \mathrm{~km} \mathrm{~s}^{-1}$, was discovered by Brown et al. (2005). Subsequent surveys have discovered dozens of further candidates. The recent review paper of Brown (2015) gives a summary, while there are subsequent papers by Savcheva et al. (2014), Theissen \& West (2014), Favia et al. (2015), Hawkins et al. (2015), Kunder et al. (2015), Li et al. (2015), and Vickers et al. (2015). It was noticed early on that the candidate HVSs exhibited significant spatial anisotropy on the sky with 8 out of the 14 HVSs at that time being located in two constellations despite a fifth of the sky having been surveyed (Brown et al. 2009b).

It is a natural extension of the Hills mechanism to ask where else it may occur. For instance, Lu et al. (2007) and Sherwin et al. (2008) suggest the central MBHs of M31 and M32 as possible sites. The recent discovery of a red supergiant runaway candidate of M31 by Evans \& Massey (2015) suggests these two possibilities may soon be testable. The Large Magellanic Cloud (LMC) is the most massive of the MW's dwarf satellites and thus there is the potential for the LMC to host a central MBH. The Hills mechanism could accelerate large numbers of stars to a few $100 \mathrm{~km} \mathrm{~s}^{-1}$ above the escape speed from the LMC. The LMC has an orbital velocity of $378 \mathrm{~km} \mathrm{~s}^{-1}$ (van der Marel \& Kallivayalil 2014); thus, alignment of these velocity vectors could result in HVSs that have been ejected from the LMC and are traveling at the escape speed of the MW. The location of the LMC on the sky and orientation of its velocity vector could explain the spatial anisotropy in the observed HVSs as suggested by Figure 1.

\section{METHOD}

To test the hypothesis that there is a significant number of HVSs ejected from the LMC, we require a prediction for the observables of such a population and a reference population of HVSs from the Galactic center (GC). We consider three models: HVGC is a model that produces stars from the GC and is identical to HV3 from Kenyon et al. (2014), HVLMC-A is a population from the LMC, and HVLMC-B is identical to HVLMC-A but has the initial ejection velocity cut by $200 \mathrm{~km} \mathrm{~s}^{-1}$ to approximate the escape velocity from the center of the LMC. All three models consider $3 M_{\odot}$ HVSs, which have a typical main-sequence lifetime of $350 \mathrm{Myr}$ since there is a large sample of B-type stars in the HVS literature we can compare to (Brown 2015). We simulate the process of ejection from the center of the LMC by seeding HVSs along the orbit of the LMC over the past $350 \mathrm{Myr}$, with a velocity that is the sum of the orbital velocity of the LMC and the ejection velocity due to the Hills mechanism. These stars are then evolved through a potential model of the MW until a randomly selected observation time, at which point we record the position and velocity in the galactic rest frame. To allow for crosscomparisons with Kenyon et al. (2014), we assume that the Sun is at a position $(x, y, z)=\left(-R_{\odot}, 0,0\right)$ relative to the GC where $R_{\odot}=8 \mathrm{kpc}$, and is traveling with a velocity $\left(v_{x}, v_{y}, v_{z}\right)=\left(0, v_{\odot}, 0\right)$ where $v_{\odot}=235 \mathrm{~km} \mathrm{~s}^{-1}$.

\subsection{LMC Black Hole Mass}

Observations of dwarf galaxy hosts of MBHs have recently been carried out by Reines et al. (2013), who specifically reference these galaxies as being in the mass range of the LMC. For dwarf galaxies with stellar masses in the range $10^{8.5} \lesssim$ $M_{\text {star }} \lesssim 10^{9.5} M_{\odot}$ they find $\mathrm{MBH}$ masses of $10^{5} \lesssim M_{\mathrm{bh}} \lesssim$ $10^{6} M_{\odot}$, with a median of $M_{\mathrm{bh}} \sim 2 \times 10^{5} M_{\odot}$. Since the dependence of the mean of the ejection velocity from the Hills mechanism is only weakly dependent on the MBH mass $\left(\propto M_{\mathrm{bh}}^{1 / 6}\right)$, see Section 2.2, a factor of 10 in MBH mass is only a factor of $\sim 1.5$ in the mean ejection velocity. Using Equation (4) from Section 2.2 , we see that the mean ejection velocity for the binaries with a separation of $0.115 \mathrm{au}$ is $\approx 1800 \mathrm{~km} \mathrm{~s}^{-1}$; thus, MBHs which are $10^{4}$ times less massive are still capable of ejecting HVSs at velocities of $\geqslant 350 \mathrm{~km} \mathrm{~s}^{-1}$. We thus assume a fiducial value $M_{\mathrm{bh}}=1.7 \times 10^{5} M_{\odot}$, similar to the median of Reines et al. (2013). 


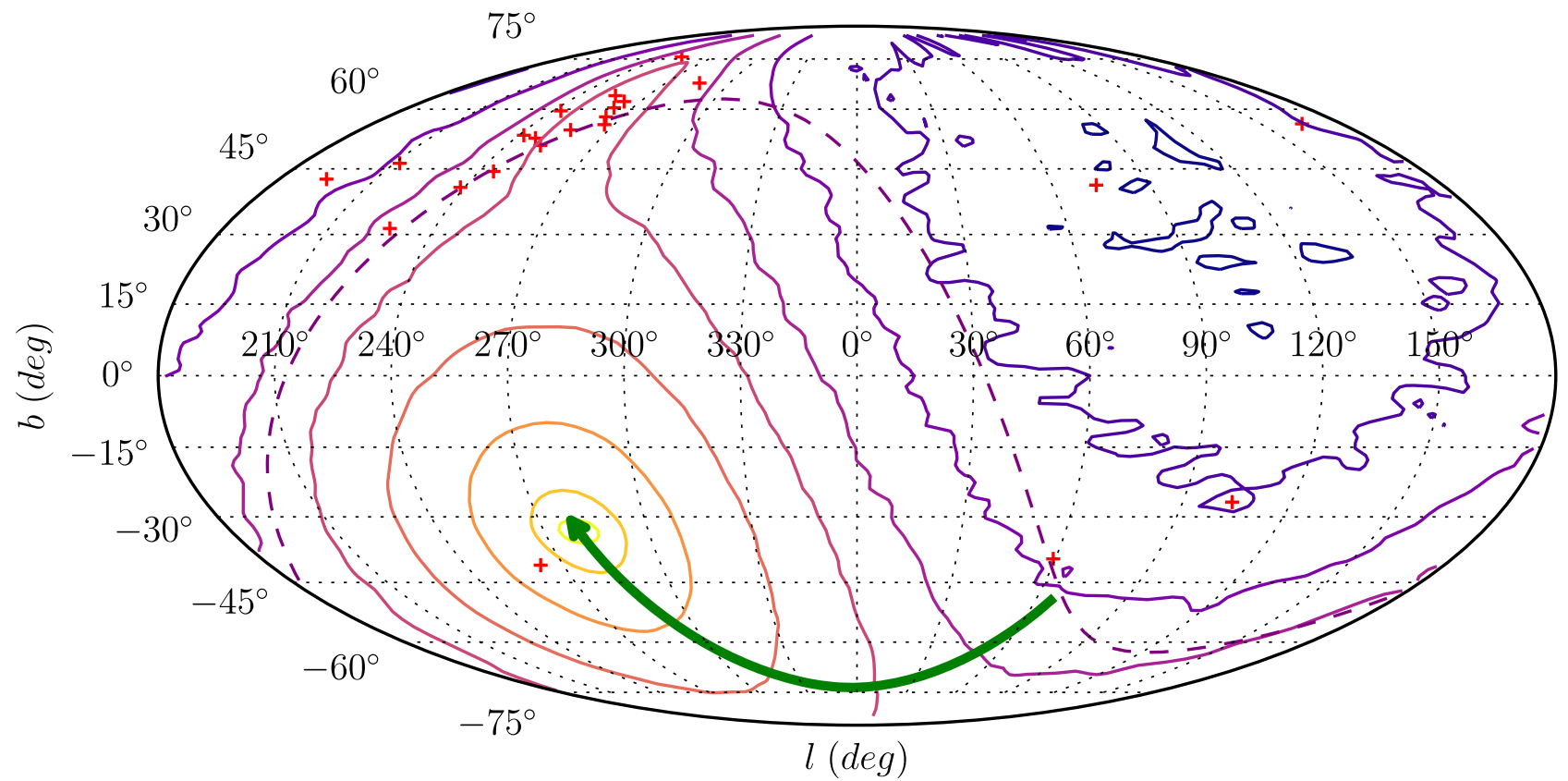

Figure 1. Predicted distribution in galactic coordinates of HVSs from the LMC at a heliocentric distance $50<d<120 \mathrm{kpc}$, using the HVLMC-A model from Section 2. Density contours are spaced $10^{1 / 2}$ apart with the yellow contour denoting the highest density, the green arrow marks the path of the LMC over the last 350 Myr, the purple dashed line is the celestial equator, and the red crosses are the HVS candidates denoted HVS1, HVS2, etc., by Brown.

\subsection{Hills Mechanism}

We first require the initial positions $\widetilde{\boldsymbol{r}}_{0}$ and velocities $\widetilde{\boldsymbol{v}}_{0}$ of the candidate HVSs in a frame co-moving with the MBH from which they were ejected. This section uses the prescription of Kenyon et al. (2014), itself based on the Hills mechanism algorithm of Bromley et al. (2006), which we briefly describe here to aid clarity. At the time of their ejection the stars are uniformly distributed across the surface of a sphere with radius $1.4 \mathrm{pc}$ centered on the MBH and given an initial outwardly radial trajectory. Thus, the only free parameters not yet determined are the magnitude of the velocity $v_{\mathrm{ej}}$, the age of the star when it is ejected $t_{\mathrm{e}}$, and the age of the star when it is observed $t_{\text {obs }}$.

Suppose that an equal mass binary with separation $a_{\text {bin }}$ interacts at a closest approach $r_{\text {close }}$ with an MBH of mass $M_{\mathrm{bh}}$. One star of the system will be ejected by the Hills mechanism with probability

$$
P_{\text {ej }}=1-D / 175 \text {, }
$$

where

$$
D=D_{0}\left(\frac{r_{\text {close }}}{a_{\text {bin }}}\right), \quad D_{0}=\left[\frac{2 M_{\mathrm{bh}}}{10^{6}\left(M_{1}+M_{2}\right)}\right]^{-1 / 3} \text {. }
$$

The probability $P$ that a star is ejected is drawn from a uniform distribution between 0 and 1 . If the star is ejected, then following Kenyon et al. (2014), the velocity is drawn from the distribution

$$
P\left(v_{\mathrm{ej}}\right) d v_{\mathrm{ej}} \propto \exp \left(-\frac{\left(v_{\mathrm{ej}}-v_{\mathrm{ej}, \mathrm{H}}\right)^{2}}{2 \sigma_{v}^{2}}\right) d v_{\mathrm{ej}},
$$

where $\sigma_{v}=0.2 v_{\mathrm{ej}, \mathrm{H}}$ and $v_{\mathrm{ej}, \mathrm{H}}$ is given by

$$
\begin{aligned}
v_{\mathrm{ej}, \mathrm{H}}= & 1760\left(\frac{a_{\mathrm{bin}}}{0.1 \mathrm{au}}\right)^{-1 / 2}\left(\frac{M_{1}+M_{2}}{2 M_{\odot}}\right)^{1 / 3} \\
& \times\left(\frac{M_{\mathrm{bh}}}{3.5 \times 10^{6} M_{\odot}}\right)^{1 / 6} f_{\mathrm{R}} \mathrm{km} \mathrm{s}^{-1} .
\end{aligned}
$$

We only consider equal mass binaries so $M_{1}=M_{2}$. Note that $f_{\mathrm{R}}$ is a normalization factor (Kenyon et al. 2014)

$$
\begin{aligned}
f_{\mathrm{R}}= & 0.774+\left(0.0204+\left(-6.23 \times 10^{-4}+\left(7.62 \times 10^{-6}\right.\right.\right. \\
& \left.\left.\left.+\left(-4.24 \times 10^{-8}+8.62 \times 10^{-11} D\right) D\right) D\right) D\right) D .
\end{aligned}
$$

Since we are interested in all possible equal mass binary systems that can result in an ejection, we sample from distributions of $a_{\text {bin }}$ and $r_{\text {close }}$. The separation of the binary $a_{\text {bin }}$ is drawn from

$$
P\left(a_{\text {bin }}\right) d a_{\text {bin }} \propto \frac{1}{a_{\text {bin }}} d a_{\text {bin }},
$$

where $a_{\mathrm{bin}, \min }=0.115$ au for stars of mass $3 M_{\odot}$ and $a_{\text {bin,max }}=4 \mathrm{au}$. The closest approach $r_{\text {close }}$ has

$$
P\left(r_{\text {close }}\right) d r_{\text {close }} \propto r_{\text {close }} d r_{\text {close }},
$$

where $r_{\text {close, } \min }=1$ au and $r_{\text {close, } \max }=175 a_{\text {bin, } \max } / D_{0}$.

Both the age of the star at the time of ejection $t_{\mathrm{ej}}$ and observation $t_{\mathrm{obs}}$ are drawn from the uniform distribution

$$
P(t) d t \propto d t,
$$

where $t_{\min }=0 \mathrm{Gyr}$ and $t_{\max }=t_{\mathrm{ms}}$ and the main-sequence lifetime $t_{\mathrm{ms}}=0.35 \mathrm{Gyr}$ for stars of mass $3 M_{\odot}$. The time of 
flight of the star from ejection to observation is then defined by $t_{\mathrm{f}}=t_{\mathrm{obs}}-t_{\mathrm{ej}}$.

We discard any sampled star that does not satisfy $P_{\mathrm{ej}} \geqslant P$, $v_{\mathrm{ej}} \geqslant v_{\mathrm{ej}, \mathrm{min}}$, and $t_{\mathrm{ej}}<t_{\mathrm{obs}}$. Note that $v_{\mathrm{ej}, \mathrm{min}}$ is chosen to minimize the computation time spent on stars that will be difficult to observe. For the HVGC model this corresponds to stars that will remain in the GC so $v_{\mathrm{ej}, \mathrm{min}}=600 \mathrm{~km} \mathrm{~s}^{-1}$. For stars in the HVLMC-A and HVLMC-B models, we choose $v_{\text {ej,min }}=200 \mathrm{~km} \mathrm{~s}^{-1}$ to ensure we only include stars that escape the LMC.

\subsection{Large Magellanic Cloud Orbit}

For stars ejected from the center of the MW, the position $\boldsymbol{r}_{0}$ and velocity $\boldsymbol{v}_{0}$ vectors in the galactic rest frame satisfy $\boldsymbol{r}_{0}=\widetilde{\boldsymbol{r}}_{0}$ and $\boldsymbol{v}_{0}=\widetilde{\boldsymbol{v}}_{0}$. However, for stars ejected from the LMC

$$
\boldsymbol{r}_{0}=\widetilde{\boldsymbol{r}}_{0}+\boldsymbol{r}_{\mathrm{LMC}}\left(t_{\mathrm{f}}\right), \boldsymbol{v}_{0}=\widetilde{\boldsymbol{v}}_{0}+\boldsymbol{v}_{\mathrm{LMC}}\left(t_{\mathrm{f}}\right),
$$

where $r_{\mathrm{LMC}}\left(t_{\mathrm{f}}\right)$ and $\boldsymbol{v}_{\mathrm{LMC}}\left(t_{\mathrm{f}}\right)$ are the position and velocity vectors of the LMC $t_{\mathrm{f}}$ years ago.

These definitions require knowledge of the position and velocity of the LMC over the past $350 \mathrm{Myr}$, for which we use an orbit of the LMC around the MW taken from Jethwa et al. (2016), who rewound the orbit of the LMC from the present position including the effect of dynamical friction. The potential used by Jethwa et al. (2016) for the MW is a Navarro et al. (1996, hereafter NFW) halo with mass $10^{12} M_{\odot}$ and concentration 7.328 and a Miyamoto \& Nagai (1975, hereafter $\mathrm{MN}$ ) disk with radial scale-length $3 \mathrm{kpc}$ and vertical scalelength $0.28 \mathrm{kpc}$ and a mass chosen to give the solar circular velocity at the position of the Sun. The LMC has an NFW halo with mass $2 \times 10^{10} M_{\odot}$ and concentration 9.450 and a Plummer bulge with scale-length $3 \mathrm{kpc}$ and a mass chosen to satisfy the mass constraint of van der Marel \& Kallivayalil (2014).

\subsection{Milky Way Potential}

For the MW, we use the potential from Kenyon et al. (2008), which was optimized to reproduce the galactic potential at both parsec scales near the MBH Sgr $\mathrm{A}^{*}$ and at scales of tens of kiloparsecs in the halo. This potential consists of four components:

$$
\phi_{\mathrm{G}}=\phi_{\mathrm{bh}}+\phi_{\mathrm{b}}+\phi_{\mathrm{d}}+\phi_{\mathrm{h}}
$$

where

$$
\phi_{\mathrm{bh}}(r)=-\mathrm{GM}_{\mathrm{bh}} / r
$$

is the Keplerian potential of the central $\mathrm{MBH}$ with $M_{\mathrm{bh}}=3.5 \times$ $10^{6} M_{\odot}$;

$$
\phi_{\mathrm{b}}(r)=-\mathrm{GM}_{\mathrm{b}} /\left(r+a_{\mathrm{b}}\right)
$$

is the Hernquist (1990) potential of the bulge with $M_{\mathrm{b}}=3.76 \times$ $10^{9} M_{\odot}$ and $a_{\mathrm{b}}=0.1 \mathrm{kpc}$;

$$
\phi_{\mathrm{d}}(R, z)=-\mathrm{GM}_{\mathrm{d}} / \sqrt{R^{2}+\left[a_{\mathrm{d}}+\left(z^{2}+b_{\mathrm{d}}^{2}\right)^{1 / 2}\right]}
$$

is the $\mathrm{MN}$ potential of the disk with $M_{\mathrm{d}}=6 \times$ $10^{10} M_{\odot}, a_{\mathrm{d}}=2.75 \mathrm{kpc}$, and $b_{\mathrm{d}}=0.3 \mathrm{kpc}$; and

$$
\phi_{\mathrm{h}}(r)=-\mathrm{GM}_{\mathrm{h}} \ln \left(1+r / r_{\mathrm{h}}\right) / r
$$

is the NFW potential of the dark matter halo with $M_{\mathrm{h}}=$ $10^{12} M_{\odot}$ and $r_{\mathrm{h}}=20 \mathrm{kpc}$. The values for each parameter are from Kenyon et al. (2014).

\section{DISCUSSION}

\subsection{All-sky Density Plots}

The density distribution of HVSs can be illustrated by an allsky plot in galactic coordinates, using the equal-area Mollweide projection. We consider only stars with heliocentric distance $50<d<120 \mathrm{kpc}$, since the distance of the LMC is $50.1 \pm 2.5 \mathrm{kpc}$ (van der Marel \& Kallivayalil 2014) and this range covers the well-known clustering of HVSs near the constellations of Leo and Sextans reported by Brown et al. (2009b); however, the kinematics in the distance bin $0<d<50 \mathrm{kpc}$ are broadly similar. Figure 2 suggests that the expected distribution of LMC HVSs on the sky is a dipole, which contrasts with the monopole shown by HVSs from the GC. Note that for the HVGC model the first few contours are not shown since there are HVSs from the GC in our population across the entire sky.

We emphasize that the density contours are not normalized to the ejection rate from either MBH. Brown (2015) justifies an ejection rate of $10^{-4} \mathrm{yr}^{-1}$ for the $\mathrm{MW} \mathrm{MBH}$, but the ejection rate from a $\mathrm{MBH}$ at the center of the $\mathrm{LMC}$ will depend significantly on the internal dynamics and star formation rate of the LMC as well as the mass of the MBH. We postpone these considerations to a companion paper, but speculate that the large contribution of the orbital velocity makes an observable signal of LMC HVSs plausible.

In the discovery paper of HVS11, Brown et al. (2009a) commented that that star was within 3.9 of the Sextans dwarf galaxy while the typical angular separation of HVSs from Local Group dwarf galaxies is $10^{\circ}-20^{\circ}$. Brown et al. (2009a) ruled out an association with Sextans based on the $260 \mathrm{~km} \mathrm{~s}^{-1}$ relative velocity of HVS11 towards Sextans; however, the initial instinct that coincidence on the sky is a requirement for association is challenged by Figure 2. The LMC HVSs are distributed across a wide region of the sky.

Figure 1 highlights an intriguing extension of the distribution that leads the LMC and is coincident with the clump observed by Brown et al. (2009b). This is the only area of the sky that is both densely populated in our LMC HVSs models and well covered by HVS surveys since almost all of these surveys have covered solely the northern hemisphere, partially due to the footprint of SDSS. The one HVS plotted in Figure 1 that lies in the southern celestial hemisphere is HE 0437-5439, which was discovered by Edelmann et al. (2005), who noted that the flight time was longer than the main-sequence lifetime for the star, and hence either it is a blue straggler and was ejected as a binary from the GC or has its origin in the LMC.

Brown et al. (2009b) discuss previous attempts to explain the anisotropy, including selection effects, contamination by runaway companions to supernovae, tidal debris from disruption of a dwarf galaxy, temporary intermediate-mass black hole companions to the central $\mathrm{MBH}$ of the $\mathrm{MW}$, and interactions between two stellar disks near the central $\mathrm{MBH}$ that imprint their geometry on the ejected HVSs. Brown (2015) states that the anisotropy is still unexplained and that it will require a southern hemisphere survey to resolve the issue.

\subsection{All-sky Velocity Plots}

In Figure 3, we show contours of the mean heliocentric radial velocity in each bin, where the standard deviation in each plot is a couple $100 \mathrm{~km} \mathrm{~s}^{-1}$. The remaining two velocity dimensions are summarized in Figure 4, where we plot 


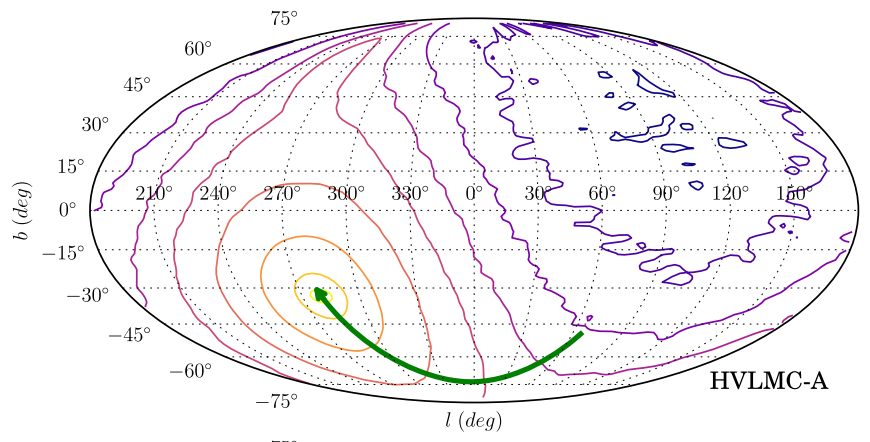

contours of the mean proper motion in each bin in both equatorial and galactic coordinates with a typical standard deviation of $0.5 \mathrm{mas}^{-1}$. For the HVLMC-A model the contours demonstrate the imprint of the orbital velocity of the LMC in the kinematics of the HVSs, while for the HVGC stars the dominant effect is the solar rotation.

Considering Figures 3 and 4, we see that at the locations on the sky of the currently observed B-type HVSs the velocity distributions of HVSs in the HVGC and HVLMC-A models are coincident. Since each model has a standard deviation of $0.5 \mathrm{mas} \mathrm{yr}^{-1}$ and the current proper motions for these stars

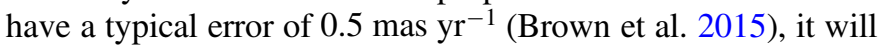
not be possible to test our hypothesis using the current sample of HVSs. This situation should be resolved by Gaia, which will give proper motions for HVSs in regions on the sky where the two models differ by up to 0.7 mas $^{-1} r^{-1}$. To illustrate this point, the first and third quartile ranges of the magnitude of the proper motions for stars at a distance $50<d<75 \mathrm{kpc}$ are $0.53<\mu<0.82$ mas yr$^{-1}$ in the HVGC model and

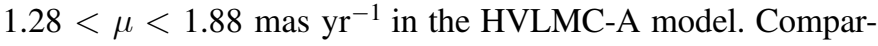
ing these ranges to end-of-mission proper motion errors for

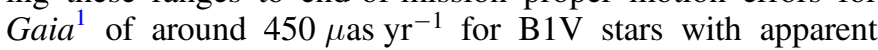
magnitude $V=20 \mathrm{mag}$, corresponding to stars at about $100 \mathrm{kpc}$, it may be possible for Gaia to distinguish between the two populations.

\subsection{Spherical Harmonics}

One method of quantifying the relative spatial distributions of HVSs from the center of the galaxy and the LMC is to consider the power in each mode of the spherical harmonic power spectrum of the density. For spherical harmonics defined by

$$
Y_{\ell}^{m}(\theta, \varphi)=\sqrt{\frac{(2 \ell+1)}{4 \pi} \frac{(\ell-m) !}{(\ell+m) !}} P_{\ell}^{m}(\cos \theta) e^{-i m \varphi},
$$

Figure 2. Density contours in galactic coordinates for the stars from the three models with heliocentric distance $50 \leqslant d \leqslant 120 \mathrm{kpc}$.

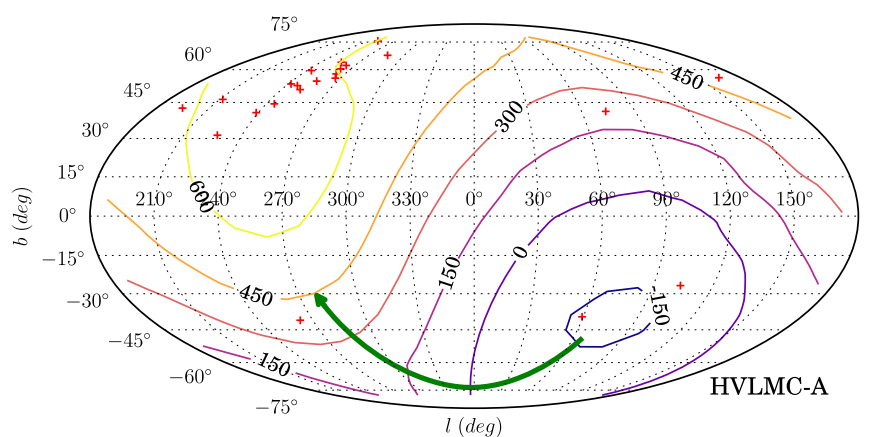

where $\theta$ and $\varphi$ are the colatidudinal and longitudinal coordinates, we can expand any function $f(\theta, \varphi)$ that is square-integrable on the unit sphere as

$$
f(\theta, \varphi)=\sum_{\ell=0}^{\infty} \sum_{m=-\ell}^{\ell} f_{\ell}^{m} Y_{\ell}^{m}(\theta, \varphi) .
$$

Using the orthonormality property of our chosen definition of the spherical harmonics, we can then write

$$
f_{\ell}^{m}=\int_{0}^{2 \pi} d \varphi \int_{0}^{\pi} d \theta \sin \theta f(\theta, \varphi) Y_{\ell}^{m *}(\theta, \varphi) .
$$

For our purposes, the function $f$ is a sum of delta functions on the unit sphere at the locations $\left(l_{i}, b_{i}\right)$ of each of the HVSs:

$$
f(b, l)=\sum_{i} \delta\left(b-b_{i}, l-l_{i}\right)
$$

Noting that $b=\frac{\pi}{2}-\theta, l=\varphi$, this sum of delta functions transforms Equation (17) into a sum over the HVSs,

$$
f_{\ell}^{m}=\sum_{i} \cos b_{i} Y_{\ell}^{m *}\left(\frac{\pi}{2}-b_{i}, l_{i}\right) .
$$

Figure 3. Mean heliocentric radial velocity for stars at a distance of $50<d<120 \mathrm{kpc}$.

\footnotetext{
1 http://www.cosmos.esa.int/web/gaia/science-performance
} 

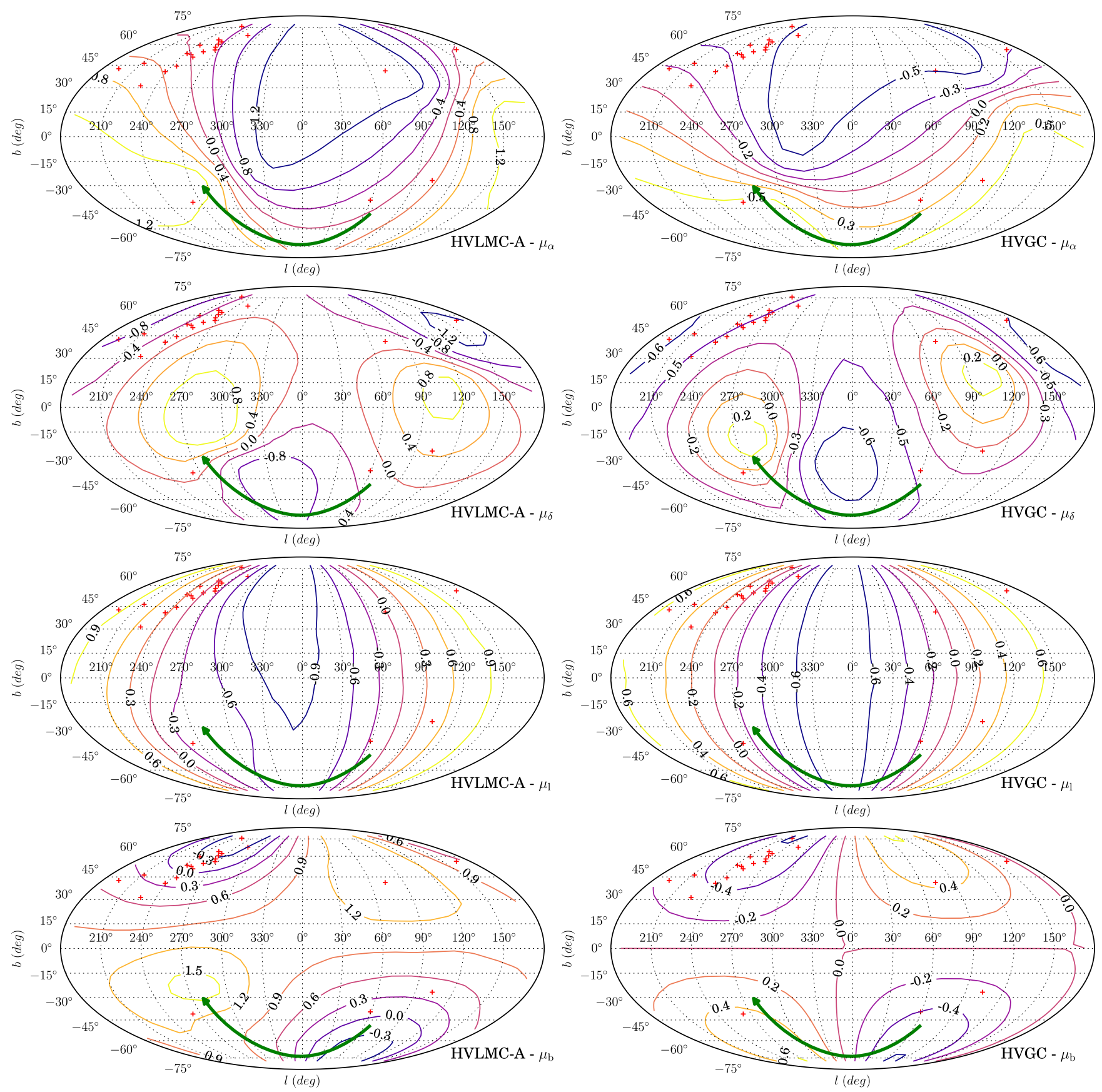

Figure 4. Mean equatorial proper motions $\left(\mu_{\alpha}, \mu_{\delta}\right)$ and galactic proper motions $\left(\mu_{1}, \mu_{\mathrm{b}}\right)$ for stars at a distance of $50<d<120 \mathrm{kpc}$.

The angular power spectrum of $f$ is then given by

$$
S(\ell)=4 \pi \sum_{m=-\ell}^{\ell}\left|f_{\ell}^{m}\right|^{2}
$$

and are plotted for our populations of HVSs in Figure 5.

Almost all of the power for the HVGC stars in this distance range is in the monopole, which we expect as the highest-velocity stars are ejected along essentially straight lines from the GC, and at $d>50 \mathrm{kpc}$ the difference between the the observer being located at the Sun or the GC is minimal. The HVLMC-A model has a peak in power in the dipole/quadrupole, with a long tail that is caused by the fast stars ejected ahead of the LMC and slow stars lagging behind. The distinguishing features between the two models are:

1. A strong monopole in the HVGC model versus a strong dipole/quadrupole in the HVLMC-A model; and

2. A large amount of power at high $\ell$ in the HVLMC-A model.

The HVLMC-B model has a large section of the sky where there are no HVSs; thus, it is not well approximated by a dipole. The power is then spread across a large number of modes since no one mode is a good approximation. With the upcoming first data release of Gaia we may soon be in a position to use spherical harmonic analysis to distinguish between production mechanisms of HVSs. 


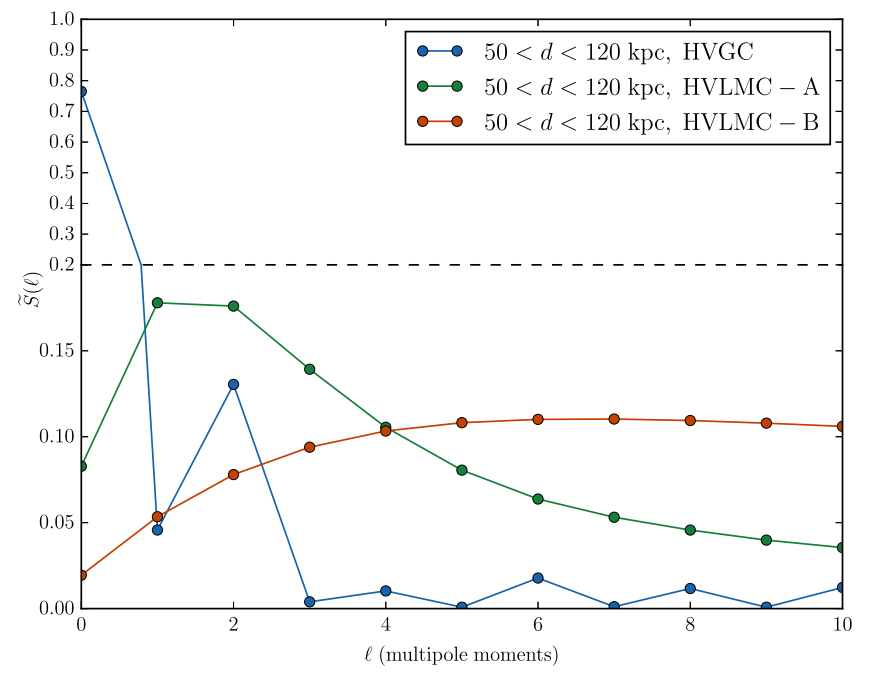

Figure 5. Spherical harmonics for the distance bin $50<d<120 \mathrm{kpc}$. The power spectrum $S(\ell)$ has been normalized by the total power for each model to give $\widetilde{S}(\ell)$ since we are primarily interested in the relative power in each mode between models.

\section{CONCLUSIONS}

HVSs created in the LMC may contribute in a significant way to the sky distribution. This may provide a natural solution to the clustering of HVSs in the direction of Leo and Sextans found by Brown et al. (2009b). Uniquely, this area of the sky is densely populated by LMC origin models and is well covered by current HVS surveys.

This hypothesis can shortly be tested by surveys in the southern celestial hemisphere, such as SkyMapper and Gaia. If these surveys fail to find a significant number of HVSs near the LMC, the model will be falsified. A possible reason for failure is that the LMC does not host a significant black hole. However, our choice of the Hills mechanism as the production route was solely due to its proposed dominance in the MW (Brown 2015). Other production routes that will be active in the disk of the LMC, such as runaway companions of supernovae and three-body dynamical interactions, can still result in highvelocity stars. Since the orbital velocity of the LMC can provide $378 \mathrm{~km} \mathrm{~s}^{-1}$ (van der Marel \& Kallivayalil 2014), stars from these two mechanisms only need to be ejected at the escape velocity of the LMC ( $2100 \mathrm{~km} \mathrm{~s}^{-1}$ ) to be considered anonymously high-velocity stars. The sky distributions of these populations are under investigation in a forthcoming paper.

The HVS candidate SDSS J121150.27+143716.2 was recently shown to be a binary by Németh et al. (2016), who concluded that the usual production routes for HVSs cannot achieve a galactic rest-frame velocity of $571.3 \mathrm{~km} \mathrm{~s}^{-1}$ without disrupting the binary, and thus the binary is either an extreme halo object or was accreted from the debris of a destroyed satellite galaxy. While the kinematics of this candidate are inconsistent with the LMC, we speculate that the addition of the orbital velocity of a Local Group dwarf galaxy with the ejection velocity due to a standard HVS production route could explain this HVS binary.

SkyMapper and Gaia will increase the quantity and quality of our HVS sample across the entire sky and thus enable more sophisticated analysis techniques, including our proposed spherical harmonic analysis, which is capable of distinguishing between hypervelocity populations in a quantitative way. Coincidence on the sky is not a necessary requirement for association with a Local Group dwarf galaxy. The satellites of the MW may well have imprinted distinctive signatures on the distribution of HVSs right across the sky.

D.B. thanks the STFC for a studentship. The orbit integration utilized galpy (Bovy 2015). We thank Prashin Jethwa for the use of his LMC orbit and Scott Kenyon for useful and prompt answers to our queries. We further acknowledge the anonymous referee for comments that improved the clarity of our results.

\section{REFERENCES}

Bovy, J. 2015, ApJS, 216, 29

Bromley, B. C., Kenyon, S. J., Geller, M. J., et al. 2006, ApJ, 653, 1194

Brown, W. R. 2015, ARA\&A, 53, 15

Brown, W. R., Anderson, J., Gnedin, O. Y., et al. 2015, ApJ, 804, 49

Brown, W. R., Geller, M. J., \& Kenyon, S. J. 2009a, ApJ, 690, 1639

Brown, W. R., Geller, M. J., Kenyon, S. J., \& Bromley, B. C. 2009b, ApJL, 690, L69

Brown, W. R., Geller, M. J., Kenyon, S. J., \& Kurtz, M. J. 2005, ApJL, 622, L33

Edelmann, H., Napiwotzki, R., Heber, U., Christlieb, N., \& Reimers, D. 2005 , ApJL, 634, L181

Evans, K. A., \& Massey, P. 2015, AJ, 150, 149

Favia, A., West, A. A., \& Theissen, C. A. 2015, ApJ, 813, 26

Hawkins, K., Kordopatis, G., Gilmore, G., et al. 2015, MNRAS, 447, 2046

Hernquist, L. 1990, ApJ, 356, 359

Hills, J. G. 1988, Natur, 331, 687

Jethwa, P., Erkal, D., \& Belokurov, V. 2016, arXiv:1603.04420

Kenyon, S. J., Bromley, B. C., Brown, W. R., \& Geller, M. J. 2014, ApJ, 793, 122

Kenyon, S. J., Bromley, B. C., Geller, M. J., \& Brown, W. R. 2008, ApJ, 680,312

Kunder, A., Rich, R. M., Hawkins, K., et al. 2015, ApJL, 808, L12

Li, Y.-B., Luo, A.-L., Zhao, G., et al. 2015, RAA, 15, 1364

Lu, Y., Yu, Q., \& Lin, D. N. C. 2007, ApJL, 666, L89

Miyamoto, M., \& Nagai, R. 1975, PASJ, 27, 533

Navarro, J. F., Frenk, C. S., \& White, S. D. M 1997, ApJ, 490, 493

Németh, P., Ziegerer, E., Irrgang, A., et al. 2016, ApJL, 821, L13

Reines, A. E., Greene, J. E., \& Geha, M. 2013, ApJ, 775, 116

Savcheva, A. S., West, A. A., \& Bochanski, J. J. 2014, ApJ, 794, 145

Sherwin, B. D., Loeb, A., \& O'Leary, R. M. 2008, MNRAS, 386, 1179

Theissen, C. A., \& West, A. A. 2014, ApJ, 794, 146

van der Marel, R. P., \& Kallivayalil, N. 2014, ApJ, 781, 121

Vickers, J. J., Smith, M. C., \& Grebel, E. K. 2015, AJ, 150, 77 